\title{
Alpha-naphthyl Butyrate Esterase
}

National Cancer Institute

\section{Source}

National Cancer Institute. Alpha-naphthyl Butyrate Esterase. NCI Thesaurus. Code C89857.

A selective cytochemical monocyte marker for alpha-naphthyl butyrate at $\mathrm{pH}$ 6.1. Alphanaphthyl butyrate covers the external surface of the plasma membrane in monocytes and membrane-bounded intracellular organelles in lymphocytes. 